\title{
Occurrence of a paroxysmal nocturnal hemoglobinuria clone in an essential thrombocythemia: a link between
} $P I G V$ and $M P L$

Paroxysmal nocturnal hemoglobinuria $(\mathrm{PNH})$ is a rare acquired hemopathy (about 1.3 individuals per million incidence) characterized by hemolytic anemia and venous thrombosis. ${ }^{1}$ The molecular defect involved is glycosylphosphatidylinositol (GPI) anchor loss related to X-linked PIGA gene mutations. Other genes responsible for the GPI anchor biosynthesis pathway could also be involved, ${ }^{2-5}$ and, research on their involvement in the pathophysiogenesis of $\mathrm{PNH}$ and its borderline forms is being uncovered.

Here, we report a case of essential thrombocythemia (ET) caused by a somatic mutation in MPL (c.1544G>T:p.W515L) shortly preceded by copy-neutral loss of heterozygosity (CN-LOH) in cis on chromosome 1, leading to homozygosity of both the acquired MPL missense mutation and an inherited heterozygous stop-gain mutation in PIGV (c.1405C>T:p.R469X) causing PNH.

\section{Clinical presentation}

In July 2017, a 73-year-old Caucasian patient was referred to the cardiology intensive care untit due to pulmonary embolism associated with cardiogenic shock. Thrombolysis was performed, followed by anticoagulant therapy. Active neoplasm and the antiphospholipid syndrome were excluded. The persistence of thrombocytosis after 3 months (platelets $>60010 \% / \mathrm{L}$ ) without any sign of infection or iron deficiency, justified a myelogram revealing ET with a clone size of $74 \%$ (Figure 1).

Concomitantly, glomerulopathy was identified (urinary protein to creatinine ratio $[\mathrm{UPCr}] 5.3 \mathrm{~g} / \mathrm{g}$; serum albumin $33 \mathrm{~g} / \mathrm{L}$; 39 urinary red cells $/ \mathrm{mL}$ ). Renal biopsy showed interstitial fibrosis with focal segmental glomerulosclerosis (FSGS). Causes of secondary FSGS were ruled out. Increased level of the nephrotic syndrome-associated soluble urokinase-type plasminogen activated receptor (suPAR) was identified (731 $\mathrm{pg} / \mathrm{mL}$ ). Blood tests showed hemolysis (lactate dehydrogenase 1,270 IU/L, undetectable haptoglobin) without schistocytes or positive Coombs test (Figure 1). PNH was suspected then confirmed by flow cytometry (80.4\% CD14- CD55FLAER- monocytes and 88.8\% CD24- CD16- CD55- FLAER' granulocytes) with an 88\% clone size at Q4 2017 (Figure 1).

Thus, this patient developed a massive pulmonary embolism, with two underlying clonal myeloid disorders (ET and $\mathrm{PNH})$ and a nephrotic syndrome. After 24 months of treatment, combining hydroxycarbamide $(6,500 \mathrm{mg} /$ week) and the $\mathrm{C} 5$ complement inhibitor eculizumab ( $900 \mathrm{mg} / 2$ weeks), the clones size dramatically decreased, cell counts normalized, hemolysis stopped, and uPCr decreased below $2 \mathrm{~g} / \mathrm{g}$

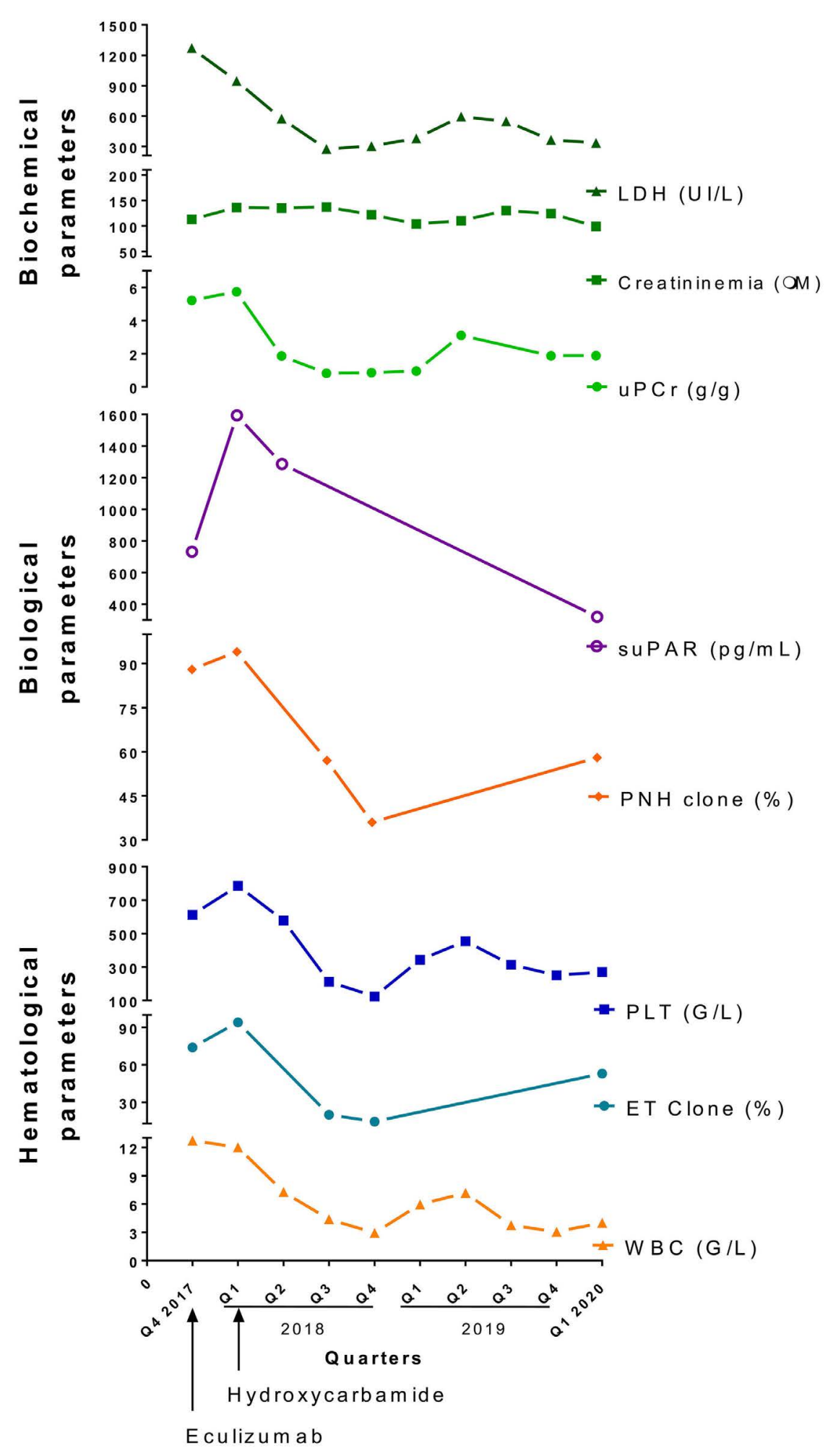

Figure 1. Follow-up over time of biochemical (LDH, creatininemia, proteinuria [urinary protein to creatinine ratio, uPCr], soluble urokinase-type plasminogen receptor [UPAR]) and hematological paroxysmal nocturnal hemoglobinuria (PNH) clone (flow cytometry), variant allele frequency (VAF) of the essential thrombocythemia (ET) clone, platelets (PLT), and leukocytes (white blood cells, WBC) parameters. Eculizumab treatment was first started in quarter 4 (Q4) of 2017, leading to a decrease in LDH Hydroxycarbamide was administered in Q1 of 2018. Apart from creatininemia, which did not vary, all other parameters followed the evolution of the $\mathrm{PNH} / \mathrm{ET}$ clone undergoing cytoreductive treatment. The concentration of serum SUPAR was assessed using the human suPAR enzyme-linked immunosorbant assay kit (Fine Test, EMELCA Biosciences) following the manufacturer's instructions. 
A

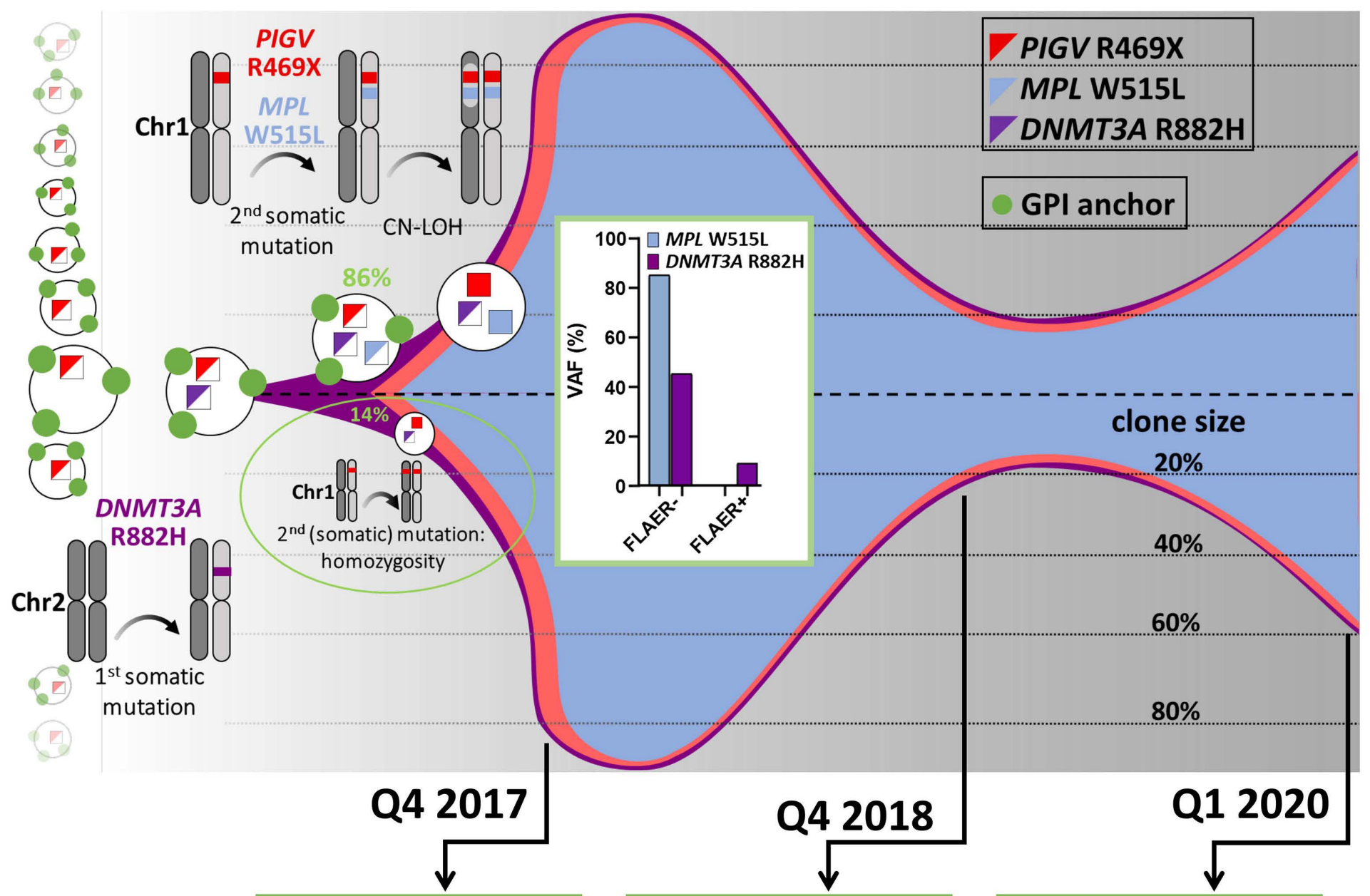

$\mathbf{B}$

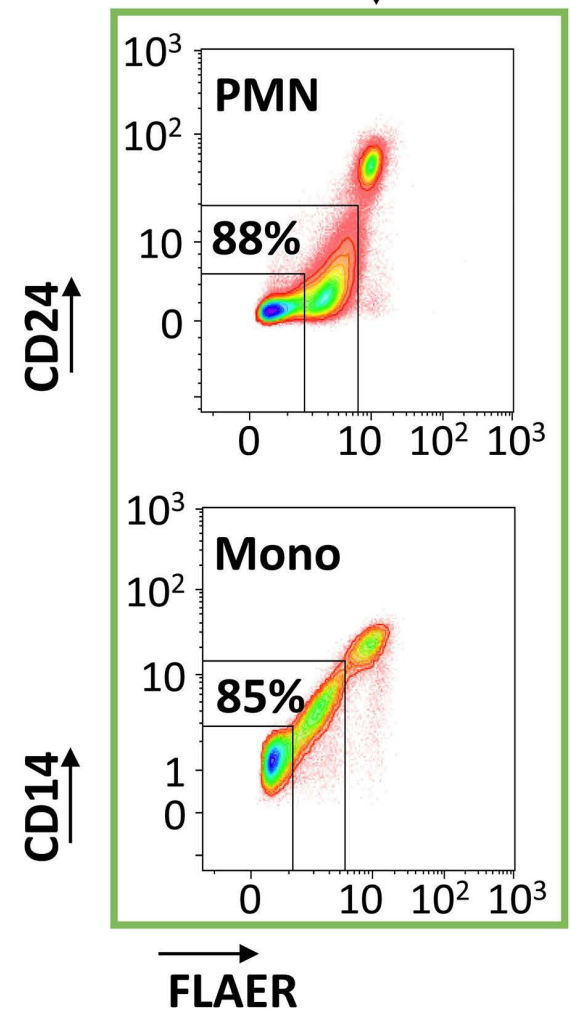

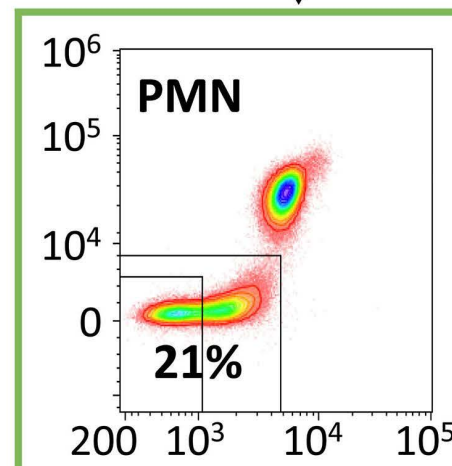

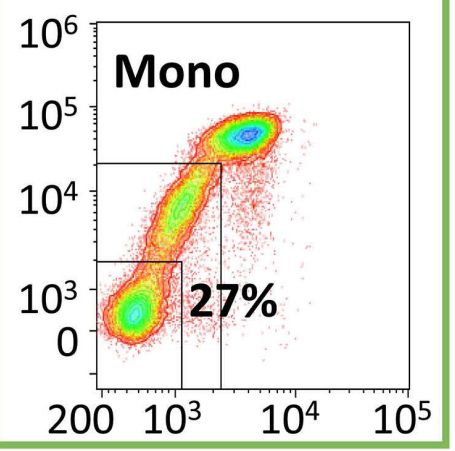

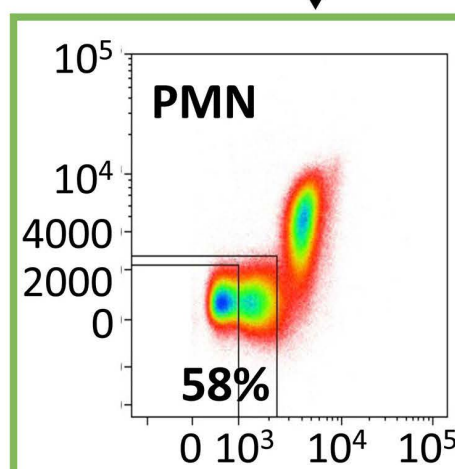

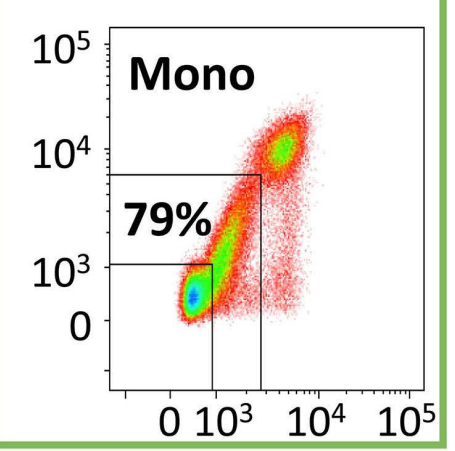

FLAER

Figure 2. About 2 years of essential thrombocythemia/paroxysmal nocturnal hemoglobinuria (ET/PNH) clone follow-up. (A) Fish diagram representation of mutation events in hematopoietic stem and progenitor cells (HSPC) and clonal expansion of the essential thrombocythemia/paroxysmal nocturnal hemoglobinuria (ET/PNH) clone over time. Heterozygous mutations are depicted as colored triangles in squares, homozygous mutations as colored squares: PIGV p.R469X red, MPL p.W515L blue, DNMT3A p.R882H purple; the GPI-anchor is depicted as green circles. ET and PNH clone size and timeline of mutation events were determined by variant allele frequency (VAF) of MPL p.W515L and DNMT3A p.R882H mutations in FLAER-positive and FLAER-negative sorted cells collected in quarter 1 (Q1) of 2018. Upon hydroxycarbamide treatment, the PNH/ET clone reduced in size reaching a low of $21 \%$ in Q4 of 2018. For cell sorting analysis, whole blood was labeled with FLAER-Alexa 488; after erythrocyte lysis, cells were resuspended in phosphate-buffered saline (PBS) and sorted with BD Influx ${ }^{\top M}$ cell sorter (BD Biosciences, Software software) (pressure $200 \mathrm{PSI}$, nozzle $100 \mu \mathrm{m}$ ) according to the gating strategy. (B) Flow cytometry follow up at Q4 of 2017, Q4 of 2018, and Q1 of 2020 of the PNH/ET clone, monitoring of neutrophil and monocyte populations. After counting, white blood cells (WBC) were incubated with pretitrated FLAER-Alexa 488 (Cedarlane Laboratories), CD16-PE (clone 3G8, Beckman Coulter), CD33-PC5.5 (clone D3HL60.251, Beckman Coulter), CD55-PE-Cy7 (clone JS11, Biolegend), CD14-APC (clone M5E2, Biolegend), CD24-APC-Vio770 (clone REA832, Miltenyi), CD15-V450 (clone HI98, BD Biosciences) and CD45-BV510 (clone HI30, BD Biosciences). After erythrocyte lysis (BD FACS Lysing Solution, BD Biosciences), cells were analyzed (Navios flow cytometer, Beckman Coulter, $>200,000$ cells/analysis). Percentages of FLAER- and GPI-anchored protein expressions (CD14, CD55 on monocytes [mono] and CD24, CD16, CD55 on polymorphonuclear neutrophils [PMN]) were analyzed using Kaluza software (monocytes gated as side scatterlow, CD45 bright, and CD33 $3^{\text {bright }}$ events; PMN gated as side scatter ${ }^{\text {high }}$, CD45 ${ }^{\text {mid }}$, and CD33 ${ }^{\text {mid }}$ events). 
A Chromosome 1

$27,124,238-27,124,270$ bp

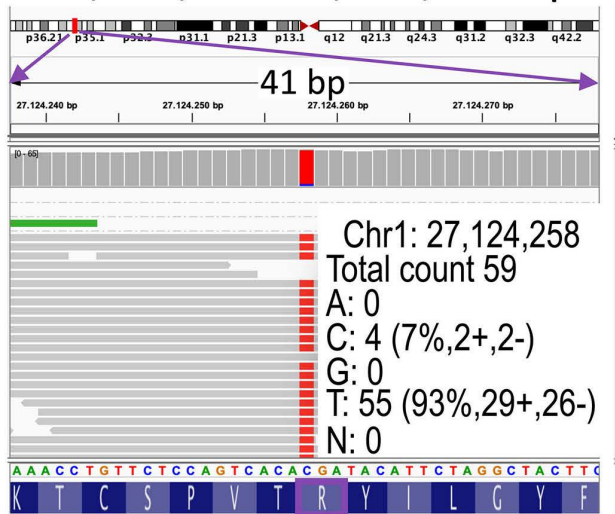

PIGV R469X
Chromosome 1 $43,814,989-43,815,029$ bp

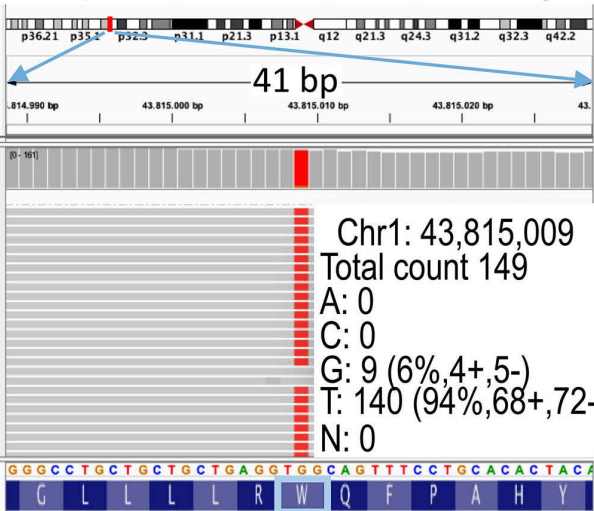

MPL W515L
Chromosome 2

$25,457,222-25,457,262 \mathrm{bp}$

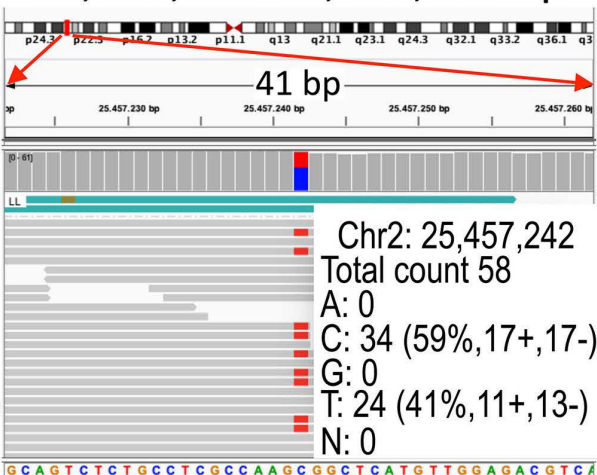

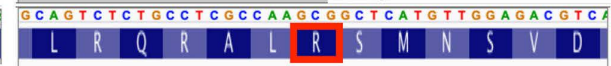

B

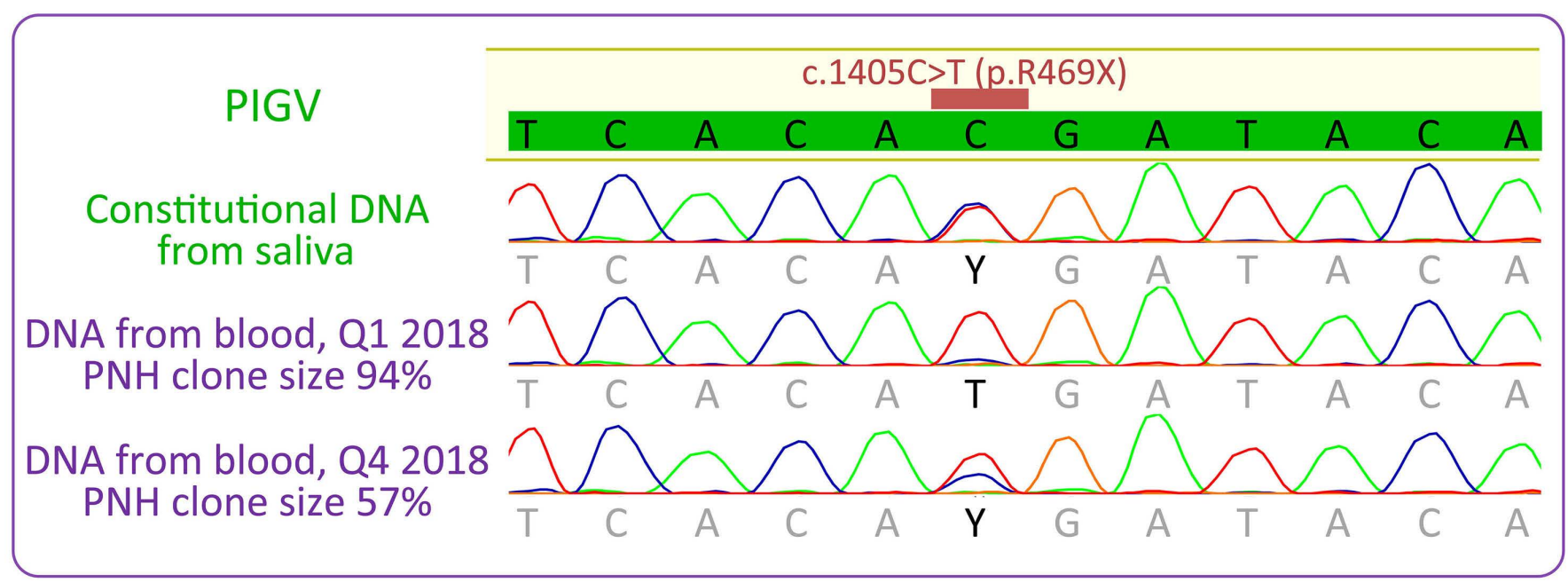

C

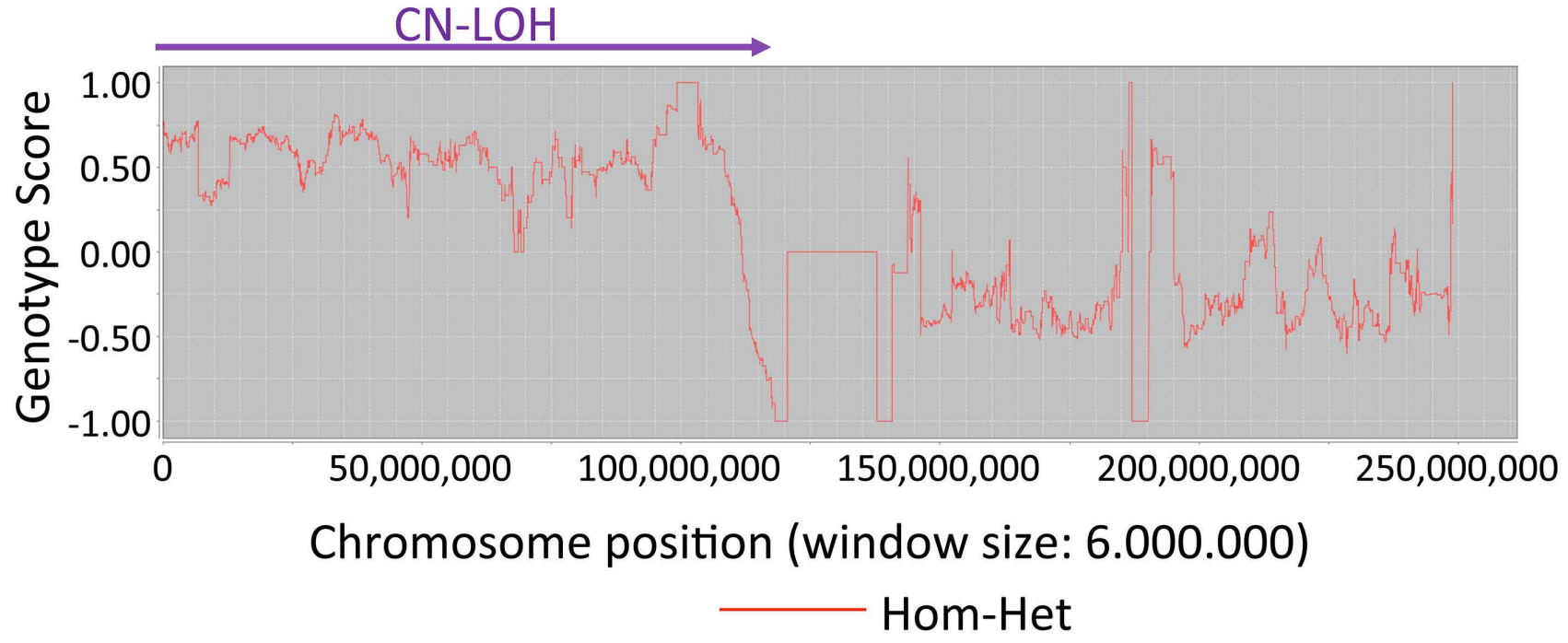

Figure 3. Illustration of the different molecular genetic explorations of the identified mutations. (A) Integrative genomic view of $41 \mathrm{bp}$ windows showing read coverage of aligned short reads from whole exome sequencing (WES) on DNA from quarter 1 (Q1) 2018 (paroxysmal nocturnal hemoglobinuria [PNH] clone sized of 94\%). Varainat allele frequency (VAF) is depicted in extra window for PIGV (R469X; chr1: 27.124.258C>T), MPL (W515L; chr1:43.815.009G>T), and DNMT3A (R882H; chr2:25.457.242C>T), hg19 reference sequence and amino acid sequence at the bottom. WES was performed using a SureSelect Human All Exon XT V6 (Agilent) enrichment kit on DNA extracted from blood samples obtained on Q1 of 2018 (PNH clone size 94\%) followed by sequencing on a HiSeq 2500 (Illumina). Reads were mapped to GRCh37 and variants were called according to GATK ${ }^{14}$ best practice guidelines. Variants were filtered and assessed based on ACMG guidelines. All mutations were confirmed by Sanger sequencing. (B) Sanger sequencing trays of PIGV gene on DNA from saliva, and DNA from two blood samples (PNH clone size $94 \%$ and $57 \%$ at Q1 and Q3 of 2018, respectively). VAF is depicted by reduced chromatogram intensity of the C allele on position c.1405. (C) HomsI ${ }^{15}$ analysis of WES data on DNA from Q1 of 2018 (PNH clone size of 94\%). From position 0 to 105,000,000 homozygous variants followed consecutively within a window size of $6 \mathrm{Mbp}$. Copy number abnormalities and copy-neutral loss of heterozygosity (CN-LOH) on the p-arm of chromosome 1. CN-LOH was confirmed by chromosomal microarray (CMA) according to manufacturer instructions using a SurePrint G3 Human CGH 4x180K oligonucleotide array (Agilent). The estimated average distance between euchromatic oligonucleotide markers was $16.1 \mathrm{~kb}$. Copy number variation (CNV) calling was performed using Cytogenomics software (vs 4.0.3.12, Agilent) using aberration algorithm ADM-2. The mosaic aberration filter was used with the following settings for deletions/losses: minimum size (kb) of the region for deletion 0.0 , the minimum number of probes for deletion $\geq 5$, minimum absolute average Log-ratio for deletion $\geq 0.15$. 
(Figure 1). He has subsequently been seen twice at 6-month intervals by teleconsultation due to the pandemic. All his hematological, cytological, and biochemical parameters were stable and proteinuria remained constant at $<1 \mathrm{~g} / \mathrm{g}$ (data not shown).

\section{Results and discussion}

In order to identify the molecular cause underlying the patient's condition targeted sequencing was performed on DNA extracted in Q4 2017 (clone size 74\%) and mutations in MPL (c.1544G>T:p.W515L) and DNMT3A (c.2645G>A:p.R882H) were discovered with a variant allele frequency (VAF) of $59 \%$ and $29 \%$, respectively. As mutations in PIGA were not found, whole exome sequencing (WES) was performed on DNA from blood extracted in Q1 2018 revealing the cause of the increased PNH clone (size 94\%): a mutation in PIGV (c.1405C>T:p.R469X) with a VAF of 93\%; concomitantly, the VAF of MPL (p.W515L) and DNMT3A (p.R882H) increased to $94 \%$ and $41 \%$ (Figure 2). Subsequent sequencing of FLAER sorted cells isolated at Q1 2018 revealed a VAF of the MPL (p.W515L) mutations of $86 \%$ and DNMT3A (p.R882H) $45 \%$ in FLAER-negative cells, while the MPL mutation p.W515L was absent in FLAER-positive cells, DNMT3A p.R882H had a VAF of $10 \%$ (Figure 2, VAF box).

Congenital heterozygosity of PIGV p.R469X was confirmed by Sanger sequencing of DNA from saliva. Homozygosity of p.R469X in PIGV and p.W515L in MPL was due to a CN$\mathrm{LOH}$ in cis of almost the entire chromosome $1 \mathrm{p}$-arm in cells derived from the mutated GPI-deficient hematopoietic stem and progenitor cells (HSPC), as confirmed by exome data and chromosomal microarray analyses (CMA) (Figure 3).

Based on the VAF of the identified mutations in cells sorted by FLAER, we can conclude on the temporal occurrence of the observed somatic mutations (Figure 2). First, an HSPC acquired a somatic mutation in DNMT3A (p.R882H) on one allele of chromosome 2, possibly a long time ago, in a stable manner and without malignant consequences, as discussed by Sun and Babushok. ${ }^{6}$ Hypomethylation of DNA due to mutated $D N M T 3 A^{7}$ probably facilitated further somatic events in this HSPC: i) homozygosity of p.R469X in PIGV giving rise to GPI-deficient cells (deriving from about $14 \%$ of GPI-deficient cells without MPL p.W515L mutation), ii) acquisition of the p.W515L mutation in MPL and iii) finally, the triple mutant HSPC with heterozygous mutations in DNTM3A (p.R882H), PIGV (p.R469X), and MPL (p.W515L) acquired CN-LOH of almost the entire $p$-arm on chromosome 1, causing homozygosity of the PIGV (p.R469X) and MPL (p.W515L) mutations leading to GPI deficiency. Immune evasion, clonal expansion, and dominance of the double mutant HSPCs led to the emergence of PIGV-PNH and ET. Interestingly, hemolysis, thrombosis, and the glomerular syndrome were observed without any signs of autoinflammation in this patient, contrary to the reports of PIGT and PIGB-PNH. ${ }^{4,5}$ Thus, we speculate that a GPI precursor with only one mannosyl group and lacking ethanolamine residues does not trigger auto-activation of the inflammasome. The ET-type myeloproliferative syndrome due to the MPL (p.W515L) mutation gave the proliferative advantage to the $\mathrm{PNH}$ clone within a mixed ET-PNH clone that explains the response to cytoreductive treatment and retrogression of PNH in the patient. Interestingly, a correlation between the clone size, the suPAR serum levels, the renal disease course, and the administration of hydroxycarbamide was observed. Although nephrotic syndrome and FSGS are not common manifestations of PNH and ET, excessive concentrations of sUPAR secreted by myeloid cells were associated with FSGS and kidney disease in human and animal models. ${ }^{8-10}$ Physiologically, the binding of UPAR to podocyte $\alpha_{v} \beta_{3}$ integrins ensures the structural stability of the podocyte cytoskeleton and its filtration properties. ${ }^{11}$ We hypothesized that the massive expansion of the GPI-deficient myeloid clones may have led to the production of the high concentration of sUPAR, which in turn may have competed with its functional GPI-anchored form, UPAR, affecting the podocyte integrity in the glomeruli, potentially causing FSGS. ${ }^{12}$

Although PIGV functions downstream of PIGA in the GPI anchor synthesis pathway, ${ }^{13}$ such a clinical course with major thrombotic events, hemolysis, and kidney failure has neither been described in a patient nor has it been associated with a GPI anchor deficiency before.

In summary, we described the first case of PIGV-PNH that emerged with an ET clone. We concluded that driving mutations of the myeloproliferative syndrome in combination with mutations in genes of the GPI anchor pathway may lead to a mixed ET-PNH clone. Finally, we showed that cytoreductive treatment in combination with administration of eculizumab led to improvement of renal and hematological outcomes.

\section{Authors}

Alexej Knaus, ${ }^{1 *}$ François Vergez, ${ }^{2 *}$ Cédric Garcia, ${ }^{3 *}$ Hartmut Engels, ${ }^{4}$ Hela Hundertmark, ${ }^{4}$ David Ribes, ${ }^{5}$ Laetitia Largeaud, ${ }^{2}$ Suzanne Tavitian, ${ }^{6}$ Bernard Payrastre, ${ }^{3}$ Peter Krawitz, ${ }^{1}$ Stanislas Faguer ${ }^{5}$ and Agnes Ribes ${ }^{3}$

${ }^{1}$ Institute for Genomic Statistics and Bioinformatics, University of Bonn, School of Medicine and University Hospital of Bonn, Bonn, Germany.; ${ }^{2}$ Department of Biological Hematology, ToulouseOncopole University Cancer Institute, Toulouse, France; ${ }^{3}$ Hematology Laboratory, Toulouse University Hospital Center, and National Referral Center for Platelets Diseases, INSERM U1297, and Paul Sabatier University, Institute of Cardiovascular and Metabolic Diseases, Toulouse, France; ${ }^{4}$ Institute of Human Genetics, University 
of Bonn, School of Medicine and University Hospital of Bonn, Bonn, Germany; ${ }^{5}$ Department of Nephrology and Organ Transplantation, Toulouse University Hospital Center, and Referral Center for Rare Renal Diseases, Toulouse University Hospital Center, Toulouse, France and ${ }^{6} \mathrm{Hematology}$ Department, Toulouse-Oncopole University Cancer Institute, Toulouse, France.

${ }^{*} A K, F V$ and $C G$ contributed equally as co-first authors.

Correspondence:

A. RIBES - ribes.a@chu-toulouse.fr

https://doi.org/10.3324/haematol.2021.279804

Received: August 20, 2021.

Accepted: January 12, 2022.

Pre-published: January 27, 2022.

(C2022 Ferrata Storti Foundation

Haematologica material is published under a CC-BY-NC license @@

\section{Disclosures}

No conflicts of interest to disclose.

\section{Contributions}

AK performed sequencing studies and analyzed the data; FV performed the cytometry analysis for PNH clone and analyzed the data; DR and ST provided patient samples and characterized the patient; $C G$ and $A R$ performed research and analyzed the data; AR designed the study, $\mathrm{HE}, \mathrm{HH}, \mathrm{BP}$ and PK participated in the discussion; AK, FV, SF, and AR wrote the paper.

\section{Acknowledgments}

The authors would like to thank Alexia Zakaroff-Girard and Elodie Riant for helpful assistance in their field of expertise, Cytometry Platform, I2MC, and the Institute of Metabolic and Cardiovascular Diseases, Toulouse, France. The authors would also like to thank Dr. Sophie Kaltenbach (Hôpital Necker-Enfants Malades, Paris, France) and Prof. Dominique Helley (Hôpital Européen Georges Pompidou, Paris, France) for their technical assistance on the molecular biology of PIGA as well as for their expert advice and gracious availability.

\section{References}

1. Brodsky RA. Paroxysmal nocturnal hemoglobinuria. Blood. 2014;124(18):2804-2811.

2. Höchsmann B, Murakami Y, Osato M, et al. Complement and inflammasome overactivation mediates paroxysmal nocturnal hemoglobinuria with autoinflammation. J Clin Invest. 2019;129(12):5123-5136.

3. Krawitz PM, Höchsmann B, Murakami Y, et al. A case of paroxysmal nocturnal hemoglobinuria caused by a germline mutation and a somatic mutation in PIGT. Blood. 2013;122(7):1312-1315.

4. Kawamoto M, Murakami Y, Kinoshita T, Kohara N. Recurrent aseptic meningitis with PIGT mutations: a novel pathogenesis of recurrent meningitis successfully treated by eculizumab. BMJ Case Rep. 2018;2018:bcr2018225910.

5. Langemeijer S, Schaap C, Preijers F, et al. Paroxysmal nocturnal hemoglobinuria caused by CN-LOH of constitutional PIGB mutation and 70-kbp microdeletion on 15q. Blood Adv. 2020;4(22):5755-5761.

6. Sun L, Babushok DV. Secondary myelodysplastic syndrome and leukemia in acquired aplastic anemia and paroxysmal nocturnal hemoglobinuria. Blood. 2020;136(1):36-49.

7. Liu X, Kamatani Y, Terao C. Genetics of autosomal mosaic chromosomal alteration (mCA). J Hum Genet. 2021;66(9):879-885.

8. Hayek SS, Leaf DE, Samman Tahhan A, et al. Soluble Urokinase receptor and acute kidney injury. $\mathrm{N}$ Engl $\mathrm{J}$ Med. 2020;382(5):416-426.

9. Shankland SJ, Pollak MR. A suPAR circulating factor causes kidney disease. Nat Med. 2011;17(8):926-927.

10. Wei C, El Hindi S, Li J, et al. Circulating urokinase receptor as a cause of focal segmental glomerulosclerosis. Nat Med. 2011;17(8):952-960.

11. Wei C, Möller CC, Altintas MM, et al. Modification of kidney barrier function by the urokinase receptor. Nat Med. 2008;14(1):55-63.

12. Sloand EM, Pfannes L, Scheinberg P, et al. Increased soluble urokinase plasminogen activator receptor (suPAR) is associated with thrombosis and inhibition of plasmin generation in paroxysmal nocturnal hemoglobinuria (PNH) patients. Exp Hematol. 2008;36(12):1616-1624.

13. Kang JY, Hong Y, Ashida $\mathrm{H}$, et al. PIG-V involved in transferring the second mannose in glycosylphosphatidylinositol. J Biol Chem. 2005;280(10):9489-9497.

14. McKenna A, Hanna M, Banks E, et al. The Genome Analysis Toolkit: a MapReduce framework for analyzing next-generation DNA sequencing data. Genome Res. 2010;20(9):1297-1303.

15. Görmez Z, Bakir-Gungor B, Sagiroglu MS. HomSI: a homozygous stretch identifier from next-generation sequencing data. Bioinformatics. 2014;30(3):445-447. 